\title{
821 MACHINE LEARNING MODELS CAN QUANTIFY CD8 POSITIVITY IN LYMPHOCYTES IN MELANOMA CLINICAL TRIAL SAMPLES
}

'Benjamin Glass*, 'S Adam Stanford-Moore, 'Diksha Meghwal, 'Nishant Agrawal, ${ }^{1}$ Mary Lin, ${ }^{1}$ Cyrus Hedvat, ${ }^{2}$ George Lee, ${ }^{2}$ Scott Ely, ${ }^{1}$ Michael Montalto, ${ }^{1}$ Ilan Wapinski, ${ }^{2}$ Vipul Baxi, 'Andrew Beck. 'PathAl, Boston, MA, United States; ${ }^{1}$ Bristol Myers Squibb, Princeton, NJ, United States

Background An accurate histological characterization of immune cells in the tumor microenvironment is essential for developing novel immune oncology targeted therapies and can assist in guiding patient treatment decisions. However, immune phenotyping is subject to challenges of manual scoring and inter-pathologist scoring variability. To support pathologistscored immune phenotyping across tumor types, we are developing machine learning (ML)-based models that can identify and quantify CD8+ lymphocytes within the stromal and parenchyma regions of tumors from non-small cell lung cancer, renal cell carcinoma, breast cancer, gastric cancer, head and neck squamous cell carcinoma, urothelial carcinoma, and melanoma. Here, we focus on the ML model for melanoma showing recent results for ML-based identification and quantification of CD8+ lymphocytes and concordance with manual pathologic assessment in data derived from clinical trials.

Methods ML algorithms were developed to quantify CD8+ lymphocytes in melanoma using 200 samples from a commercial dataset containing both primary and metastatic melanoma cases. Models were trained using the PathAI research platform on digitized whole slide images (WSI) stained for CD8 using clone C8/144b (Dako), and annotations were provided by the PathAI network of expert pathologists. Training included identification of slide artifacts, parenchyma, cancer stroma, and necrosis, as well as CD8 + lymphocytes and other CD8- cell types. Examples of melanin, such as pigmented macrophages, were added to non-CD8 + cell types. To evaluate the performance of the ML model, model-predicted CD8+ counts were compared to a consensus count from five independent pathologists for representative regions ("frames") using the Pearson correlation. This was done in 112 held-out test frames from 90 WSI baseline samples from three clinical trials of immunotherapy treatment in individuals with metastatic melanoma. Inter-pathologist agreement among the five pathologists was also calculated.

Results ML-based quantitation of CD8 positivity in lymphocytes showed high concordance with manual pathologist consensus counts. In frames validation of $\mathrm{CD} 8+$ counts on the test set of WSI, there was high correlation between the ML model and pathologist consensus counts $(r=0.92$ [95\% CI $0.88-0.94])$. This correlation was comparable to the agreement among the five expert pathologists $(r=0.88$ [95\% CI $0.85-$ 0.91]).

Conclusions ML model-predicted CD8 + cell counts are highly concordant with pathologist scores on WSI samples from melanoma-focused clinical trials. These data demonstrate the capability of AI-powered digital pathology for accurate and reproducible quantitation of $\mathrm{CD} 8+$ lymphocytes in clinical trial samples, contributing to improved evaluation of the tumor microenvironment and targeted development of therapeutics.

http://dx.doi.org/10.1136/jitc-2021-SITC2021.821 